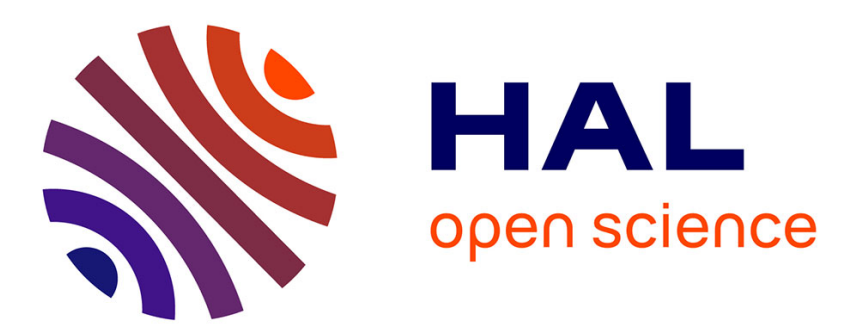

\title{
The steady oblique path of buoyancy-driven disks and spheres
}

David Fabre, Joël Tchoufag, Jacques Magnaudet

\section{To cite this version:}

David Fabre, Joël Tchoufag, Jacques Magnaudet. The steady oblique path of buoyancy-driven disks and spheres. Journal of Fluid Mechanics, 2012, vol. 707, pp. 24-36. 10.1017/jfm.2012.231 . hal00877022

\section{HAL Id: hal-00877022 \\ https://hal.science/hal-00877022}

Submitted on 25 Oct 2013

HAL is a multi-disciplinary open access archive for the deposit and dissemination of scientific research documents, whether they are published or not. The documents may come from teaching and research institutions in France or abroad, or from public or private research centers.
L'archive ouverte pluridisciplinaire HAL, est destinée au dépôt et à la diffusion de documents scientifiques de niveau recherche, publiés ou non, émanant des établissements d'enseignement et de recherche français ou étrangers, des laboratoires publics ou privés. 


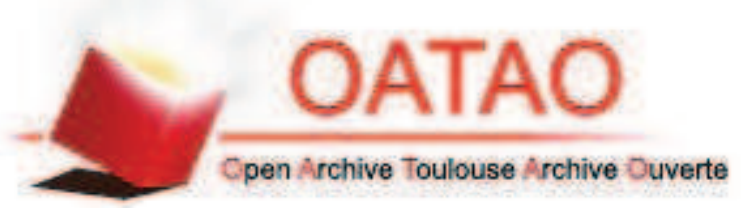

\section{Open Archive TOULOUSE Archive Ouverte (OATAO)}

OATAO is an open access repository that collects the work of Toulouse researchers and makes it freely available over the web where possible.

This is an author-deposited version published in : http://oatao.univ-toulouse.fr/ Eprints ID : 9791

To link to this article :DOI :10.1017/jfm.2012.231

URL : http://dx.doi.org/10.1017/jfm.2012.231

To cite this version Fabre, David and Tchoufag, Joël and Magnaudet, Jacques The steady oblique path of buoyancy-driven disks and spheres. (2012)

Journal of Fluid Mechanics, vol. 707. pp. 24-36. ISSN 0022-1120

Any correspondance concerning this service should be sent to the repository administrator: staff-oatao@,1istes-diff.inp-toulouse.fr 


\title{
The steady oblique path of buoyancy-driven disks and spheres
}

\author{
David Fabre $^{1}$, Joël Tchoufag ${ }^{1}$ and Jacques Magnaudet ${ }^{1,2} \dagger$ \\ ${ }^{1}$ Université de Toulouse; INPT, UPS; IMFT (Institut de Mécanique des Fluides de Toulouse); \\ Allée Camille Soula, F-31400 Toulouse, France \\ ${ }^{2}$ CNRS; IMFT; F-31400 Toulouse, France
}

We consider the steady motion of disks of various thicknesses in a weakly viscous flow, in the case where the angle of incidence $\alpha$ (defined as that between the disk axis and its velocity) is small. We derive the structure of the steady flow past the body and the associated hydrodynamic force and torque through a weakly nonlinear expansion of the flow with respect to $\alpha$. When buoyancy drives the body motion, we obtain a solution corresponding to an oblique path with a non-zero incidence by requiring the torque to vanish and the hydrodynamic and net buoyancy forces to balance each other. This oblique solution is shown to arise through a bifurcation at a critical Reynolds number $R e^{S O}$ which does not depend upon the body-to-fluid density ratio and is distinct from the critical Reynolds number $R e^{S S}$ corresponding to the steady bifurcation of the flow past the body held fixed with $\alpha=0$. We then apply the same approach to the related problem of a sphere that weakly rotates about an axis perpendicular to its path and show that an oblique path sets in at a critical Reynolds number $R e^{S O}$ slightly lower than $R e^{S S}$, in agreement with available numerical studies.

Key words: bifurcation, flow-structure interactions, wakes

\section{Introduction}

The dynamics of bodies freely falling or rising within a viscous fluid under the effect of buoyancy is currently an active field of research (see Ern et al. 2012 for a recent review). A large variety of paths has been reported, including fluttering, tumbling, spiral and chaotic motions. In some cases, such as light spheres (Jenny, Dusek \& Bouchet 2004) and thin disks with a density close to that of the fluid (Fernandes et al. 2007; Auguste 2010), a number of regimes characterized by weak deviations with respect to the vertical (collectively termed A-regimes by Ern et al.) have been noticed at Reynolds numbers significantly smaller than those for which large-amplitude oscillatory lateral motions (fluttering) are observed. The first of these non-vertical paths consists of a steady oblique (SO) trajectory, the body being slightly tilted with respect to its path (for a disk) or slowly rotating (for a sphere). In this state, the wake is characterized by the presence of a pair of steady counter-rotating streamwise vortices (Veldhuis \& Biesheuvel 2007; Horowitz \& Williamson 2010). This structure suggests that this SO path is strongly connected to the steady-state (SS) mode 
observed after the first wake bifurcation in the related problem of the flow past a fixed, non-tilted (or non-rotating) body, e.g. Fabre, Auguste \& Magnaudet (2008), Meliga, Chomaz \& Sipp (2009) and Chrust, Bouchet \& Dusek (2010). However, differences have been consistently noticed between the values of the critical Reynolds number associated with the onset of the SO path and that of the SS mode (Jenny et al. 2004; Auguste 2010). Also, it is not clear whether or not the former critical Reynolds number depends on the body-to-fluid relative density which is an additional control parameter when the body moves freely.

The goal of this paper is to derive an approximate solution for the flow around the body through a weakly nonlinear expansion of the Navier-Stokes equations, the force and torque being expanded in Taylor series with respect to the angle of incidence. We first describe the general method in the case of a slightly tilted disk of arbitrary thickness. Then we show that an equilibrium solution for a freely moving disk with non-zero incidence and non-zero inclination with respect to the vertical can be constructed, and emerges through a supercritical bifurcation (except when the disk is extremely thin, in which case the bifurcation is found to be subcritical) at a critical Reynolds number independent of the body-to-fluid density ratio. We finally apply the same approach to a sphere, the expansion then being carried out with respect to the rotation rate.

\section{A weakly nonlinear expansion for the flow past a fixed body with a small imposed incidence}

\subsection{Problem definition}

We consider a cylindrical body of diameter $d$ and thickness $h$ moving steadily at a velocity $\boldsymbol{U}_{0}$ in a quiescent viscous fluid, or equivalently the same body held fixed in a uniform flow of incoming velocity $\boldsymbol{U}_{i}=-\boldsymbol{U}_{0}$. The problem in this section is to compute the steady flow $[\boldsymbol{u}, p]$ past this body and deduce the corresponding force and torque $(\boldsymbol{F}, \boldsymbol{M})$. We define two systems of axes similar to those employed by Fabre, Assemat \& Magnaudet (2011) (see figure 1). The first of these, $(\boldsymbol{x}, \boldsymbol{y}, \boldsymbol{z})$, is associated with the body geometry, with $\boldsymbol{x}$ coinciding with the body axis. The second is the aerodynamic system $\left(\boldsymbol{x}_{a}, \boldsymbol{y}_{a}, \boldsymbol{z}\right)$, where $\boldsymbol{x}_{a}$ is aligned with the body velocity $\boldsymbol{U}_{0}$. Introducing the angle of incidence $\alpha$ between $\boldsymbol{U}_{i}$ and the body axis $\boldsymbol{x}$, one has

$$
\boldsymbol{U}_{i}=-U_{0} \boldsymbol{x}_{a}=U_{0}(\cos \alpha \boldsymbol{x}-\sin \alpha \boldsymbol{y}) .
$$

The hydrodynamic loads can also be projected onto the two systems of axes, yielding axial and lateral force components in the former and drag and lift components in the latter, with

$$
\boldsymbol{F}=-\left(D \boldsymbol{x}_{a}+L \boldsymbol{y}_{a}\right)=F_{x} \boldsymbol{x}+F_{y} \boldsymbol{y}, \quad \boldsymbol{M}=M z,
$$

the two series of force components being related through

$$
D=F_{x} \cos \alpha-F_{y} \sin \alpha, \quad L=F_{x} \sin \alpha+F_{y} \cos \alpha .
$$

The velocity field $\boldsymbol{u}$ and pressure field $p$ satisfy the steady incompressible Navier-Stokes equations which are conveniently written in the form

$$
\frac{1}{2} \mathscr{C}(\boldsymbol{u}, \boldsymbol{u})+\frac{1}{\rho} \nabla p-v \nabla^{2} \boldsymbol{u}=\mathbf{0}, \quad \nabla \cdot \boldsymbol{u}=0,
$$

where $\mathscr{C}(\boldsymbol{a}, \boldsymbol{b})=\boldsymbol{a} \cdot \nabla \boldsymbol{b}+\boldsymbol{b} \cdot \nabla \boldsymbol{a}$ is the symmetric advection operator, $\rho$ and $v$ being the fluid density and kinematic viscosity, respectively. The boundary equations to be 
(a)

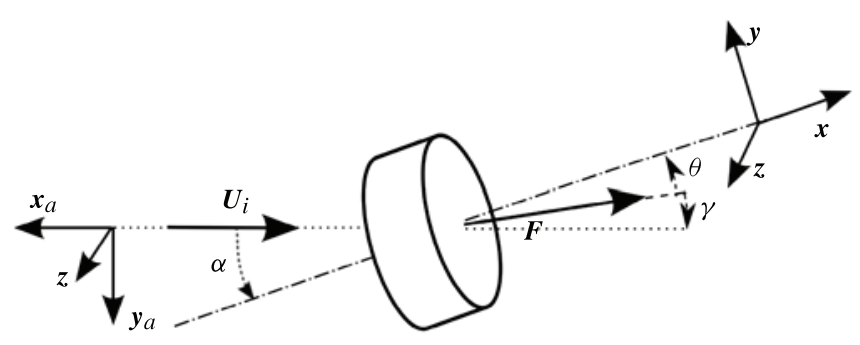

(b)

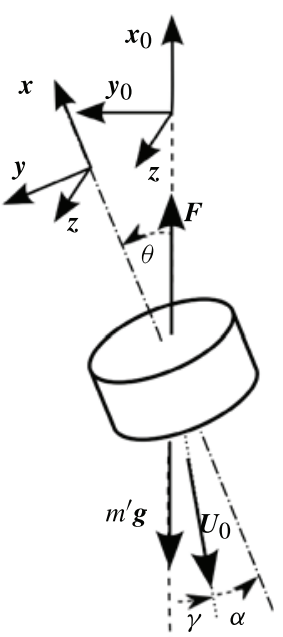

FIGURE 1. Sketch of the problem. (a) Fixed body; (b) freely moving body $\left(\left[\boldsymbol{x}_{0}, \boldsymbol{y}_{0}, \boldsymbol{z}\right]\right.$ is the laboratory system of axes and $m^{\prime} \boldsymbol{g}$ the net body weight). $\alpha$ (respectively $\theta$ ) is the angle between the incoming velocity $\boldsymbol{U}_{i}=-\boldsymbol{U}_{0}$ (respectively the hydrodynamic force $\boldsymbol{F}$ ) and the body axis $\boldsymbol{x}$, while $\gamma=\theta-\alpha$ is the angle between $\boldsymbol{F}$ and the incoming velocity ( $\gamma$ is negative in $(a)$ and positive in $(b)$ ). Note that, owing to the convention defined in (2.2), the lift component $L$ of $\boldsymbol{F}$ is positive in $(a)$ and negative in $(b)$.

satisfied by $\boldsymbol{u}$ are the no-slip condition $\boldsymbol{u}=\mathbf{0}$ at the body surface and matching with the incoming flow at infinity, i.e. $\boldsymbol{u} \rightarrow \boldsymbol{U}_{i}$ as $|\boldsymbol{R}| \rightarrow \infty, \boldsymbol{R}$ denoting the local position from the body centre of mass. Once the flow field is known, the hydrodynamic force and torque are evaluated as

$$
\boldsymbol{F}=\int_{S}\left(-p \boldsymbol{n}+\rho v\left(\nabla \boldsymbol{u}+\nabla \boldsymbol{u}^{\mathrm{T}}\right) \cdot \boldsymbol{n}\right) \mathrm{d} S, \quad \boldsymbol{M}=\int_{S} \boldsymbol{R} \times\left(-p \boldsymbol{n}+\rho v\left(\nabla \boldsymbol{u}+\nabla \boldsymbol{u}^{\mathrm{T}}\right) \cdot \boldsymbol{n}\right) \mathrm{d} S,
$$

where $\boldsymbol{n}$ is the outward unit normal to the body surface. These loads may be represented through the classical aerodynamic coefficients defined as

$$
\left[L, D, F_{x}, F_{y}\right]=\frac{\rho S U_{0}^{2}}{2}\left[C_{L}, C_{D}, C_{x}, C_{y}\right] ; \quad M=\frac{\rho S d U_{0}^{2}}{4} C_{M},
$$

where $S=\pi d^{2} / 4$ is the body cross-sectional area. The whole problem may be characterized by the Reynolds number $\operatorname{Re}=U_{0} d / v$ and the body aspect ratio $\chi=d / h$. In the rest of this section we set $U_{0}=1, d=1, \rho=1$, so that $R e=v^{-1}$.

\subsection{Weakly nonlinear expansion}

We now expand the state vector $\boldsymbol{q} \equiv[\boldsymbol{u}, p]^{\mathrm{T}}$ in the form

$$
\boldsymbol{q}=\boldsymbol{q}_{0}+\alpha \boldsymbol{q}_{\alpha}+\alpha^{2} \boldsymbol{q}_{\alpha^{2}}+\alpha^{3} \boldsymbol{q}_{\alpha^{3}}+\cdots .
$$

Injecting this ansatz into the Navier-Stokes equations results in a set of equations that must be solved at each order, along with appropriate boundary conditions. For this purpose the boundary condition at infinity (2.1) is also expanded in powers of $\alpha$, 
yielding

$$
\boldsymbol{u} \rightarrow \boldsymbol{U}_{i}=\boldsymbol{x}-\alpha \boldsymbol{y}-\frac{1}{2} \alpha^{2} \boldsymbol{x}+\frac{1}{6} \alpha^{3} \boldsymbol{y}+\cdots \quad \text { for }|\boldsymbol{R}| \rightarrow \infty .
$$

Symmetry considerations indicate that even terms in (2.7) can only result in an axial force (because the corresponding contributions do not change sign with $\alpha$ ), while odd terms only contribute to the lateral force and to the torque. Therefore the loads can be anticipated to have the form

$$
\left.\begin{array}{l}
F_{x} \approx F_{x 0}+\alpha^{2} F_{x, \alpha^{2}}+\cdots, \\
F_{y} \approx \alpha F_{y, \alpha}+\alpha^{3} F_{y, \alpha^{3}}+\cdots, \\
M \approx \alpha M_{\alpha}+\alpha^{3} M_{\alpha^{3}}+\cdots
\end{array}\right\}
$$

The numerical approach used to compute the successive terms in (2.7) is adapted from that of Meliga et al. (2009) to which the reader is referred for details. We first introduce the polar system of axes $\left(\boldsymbol{e}_{r}, \boldsymbol{e}_{\varphi}\right)$ in the $(y, z)$-plane, so as to write the velocity field in the form $\boldsymbol{u}=\left[u_{r}, u_{\varphi}, u_{x}\right]^{\mathrm{T}}$. Thanks to the modal expansion in the azimuthal direction $\varphi$, each problem then becomes two-dimensional in the $(r, x)$-plane. The finite-element FreeFem++ software is used to discretize the differential operators involved in the successive problems corresponding to the expansion (2.7). The resulting linear systems are solved with the UMFPack solver embedded in FreeFem++. A grid made of triangular elements is generated using a Delaunay-Voronoi algorithm, with local refinement at the corners of the body and in its near wake. The computational domain is a rectangle defined by $(r, x) \in\left[0, r_{\infty}\right] \times\left[x_{-\infty}, x_{\infty}\right]$ (as displayed in figure 1 of Meliga et al. 2009), where $r_{\infty}, x_{-\infty}$ and $x_{\infty}$ are chosen large enough not to have a discernible influence on the results. The boundary conditions at infinity arising from (2.8) are directly enforced at the inlet plane $\left(x=x_{-\infty}\right)$ and lateral boundary $\left(r=r_{\infty}\right)$, while a zero-traction condition is used at the outlet plane $\left(x=x_{\infty}\right)$. Details about the grid structure and the sensitivity of results to the grid density and to values of $r_{\infty}$ and $x_{\infty}$ can be found in $\S 3$ of Assemat, Fabre \& Magnaudet (2012).

The leading order in the expansion corresponds to the axisymmetric flow past a body having its axis aligned with the incoming flow. This flow satisfies the Navier-Stokes equations (2.4) with $\boldsymbol{u}_{0} \rightarrow \boldsymbol{x}$ for $|\boldsymbol{R}| \rightarrow \infty$, and is computed through a Newton iteration method. The corresponding loads are then deduced from (2.5). As expected, they reduce to an axial force (which in this case coincides with the drag), corresponding to the term $F_{x 0}$ in the expansion (2.9). The associated drag coefficient, $C_{x 0}$, is displayed in figure $2(a)$ for a disk with an aspect ratio $\chi=10$. In the range of Reynolds numbers of interest here, this coefficient is a smoothly decreasing function of $R e$ for all types of bodies.

\section{3. $O(\alpha)$ problem: linear correction due to a non-zero incidence}

The next order in the expansion (2.7) corresponds to the leading-order correction to the base flow when the body axis is slightly tilted with respect to the incoming velocity. This problem is similar to one of those considered by Fabre et al. (2011) with two-dimensional bodies, where the lift and torque coefficients $\left(L_{\alpha}, M_{\alpha}\right)$ were used to build a 'quasi-static' model relevant to freely moving bodies in the limit of large body-to-fluid density ratios. At order $\alpha$, the governing equations (2.4) and the associated far-field condition (2.8) read

$$
\mathscr{C}\left(\boldsymbol{u}_{0}, \boldsymbol{u}_{\alpha}\right)+\nabla p_{\alpha}-R e^{-1} \nabla^{2} \boldsymbol{u}_{\alpha}=\mathbf{0}, \quad \nabla \cdot \boldsymbol{u}_{\alpha}=0, \quad \boldsymbol{u}_{\alpha} \rightarrow-\boldsymbol{y} \quad \text { for }|\boldsymbol{R}| \rightarrow \infty .
$$


(a)

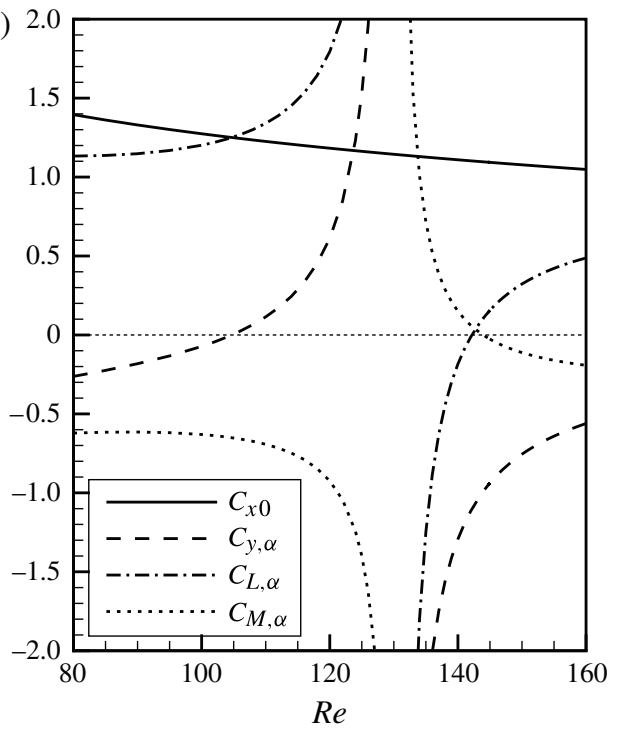

(b)

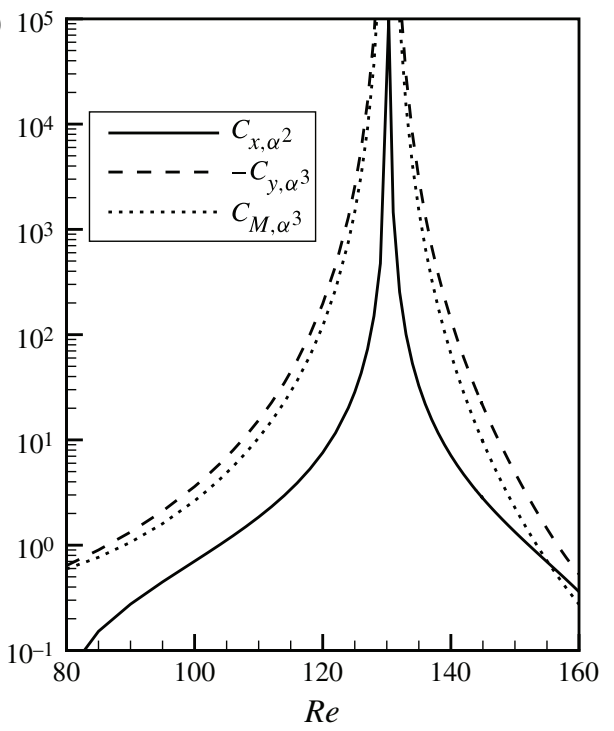

FIGURE 2. Variations with $R e$ of the coefficients entering the weakly nonlinear expansion of loads for a disk with $\chi=10$ : $(a)$ orders zero and $\alpha ;(b)$ orders $\alpha^{2}$ and $\alpha^{3}$.

We expand the state vector $\boldsymbol{q}_{\alpha}$ in the form

$$
\boldsymbol{q}_{\alpha}=\hat{\boldsymbol{q}}_{1}^{1} \mathrm{e}^{\mathrm{i} \varphi}+\overline{\hat{\boldsymbol{q}}}_{1}^{1} \mathrm{e}^{-\mathrm{i} \varphi},
$$

where $\hat{\boldsymbol{q}}_{n}^{m}$ is the complex mode of order $\alpha^{n}$ associated with an $\mathrm{e}^{\mathrm{i} m \varphi}$ azimuthal variation and the overbar denotes the complex conjugate. The mode $\hat{\boldsymbol{q}}_{1}^{1}$ is then the solution of the linear system

$$
\mathscr{A}_{1} \hat{\boldsymbol{q}}_{1}^{1}=0, \quad \hat{\boldsymbol{q}}_{1}^{1} \rightarrow[-1 / 2,-\mathrm{i} / 2,0,0]^{\mathrm{T}} \quad \text { for }|\boldsymbol{R}| \rightarrow \infty .
$$

Here $\mathscr{A}_{m}$ is the linearized Navier-Stokes operator acting on perturbations with an azimuthal modal expansion of the form $\mathrm{e}^{\mathrm{i} m \varphi}$, i.e.

$$
\mathscr{A}_{m}=\left(\begin{array}{cc}
\mathscr{C}_{m, 0}\left(\cdot, \boldsymbol{u}_{0}\right)-\operatorname{Re}^{-1} \nabla_{m}^{2} & \nabla_{m} \\
\nabla_{m}^{\mathrm{T}} & 0
\end{array}\right) .
$$

In (2.13), $\nabla_{m}$ is the gradient operator relative to the azimuthal wavenumber $m$ and $\mathscr{C}_{m, n}(\cdot, \boldsymbol{b})=\boldsymbol{b} \cdot \boldsymbol{\nabla}(\cdot)+(\cdot) \cdot \boldsymbol{\nabla} \boldsymbol{b}$ is the advection operator by which the velocity $\boldsymbol{b}$ of a mode having an azimuthal wavenumber $n$ acts on the velocity of the current mode of azimuthal wavenumber $m$, as defined in equation (C2) of Meliga et al. (2009). The solution of this linear system provides $\boldsymbol{q}_{\alpha}$ which in turn yields $F_{y, \alpha}$ and $M_{\alpha}$ through (2.5). At this order, the lift component is also linear with respect to $\alpha$, i.e. $L \approx \alpha L_{\alpha}$, and (2.3) indicates that $L_{\alpha}=F_{x 0}+F_{y, \alpha}$.

The solution of the above problem is well-defined as long as the operator $\mathscr{A}_{m}$ is not singular. As one could anticipate, the problem corresponding to $m=1$ turns out to be singular for $R e=R e^{S S}$, i.e. right at the bifurcation towards the SS wake mode. The first column of table 1 provides the numerical value of $R e^{S S}$ for disks with three different aspect ratios. The value corresponding to an infinitely thin disk $(\chi=\infty)$ is in agreement with that found in previous theoretical and computational studies (Natarajan 


\begin{tabular}{rccccccccr}
\hline$\chi$ & $R e^{S S}$ & $R e^{S O}$ & $A r^{S O}$ & $C_{x 0}^{S O}$ & $C_{x 0}^{\prime S O}$ & $C_{x, \alpha^{2}}^{S O}$ & $C_{y, \alpha}^{S O}$ & $C_{M, \alpha}^{\prime S O}$ & \multicolumn{1}{c}{$C_{M, \alpha^{3}}^{S O}$} \\
3 & 159.93 & 165.38 & 51.08 & 1.018 & -0.0025 & 9.797 & -2.051 & -0.0919 & 541.975 \\
10 & 130.30 & 143.94 & 46.15 & 1.097 & -0.0032 & 3.317 & -0.992 & -0.0267 & 13.727 \\
51 & 120.45 & 141.57 & 45.51 & 1.103 & -0.0033 & 1.871 & -0.618 & -0.0157 & 0.036 \\
52 & 120.40 & 141.56 & 45.62 & 1.103 & -0.0033 & 1.872 & -0.617 & -0.0156 & -0.013 \\
$\infty$ & 116.75 & 141.67 & 46.54 & 1.102 & -0.0033 & 1.416 & -0.512 & -0.01285 & -0.847 \\
\multirow{2}{*}{ Sphere } & $R e^{S S}$ & \multirow{2}{*}{$e^{S O}$} & $A r^{S O}$ & $C_{D 0}^{S O}$ & $C_{D 0}^{\prime S O}$ & $C_{D, \omega^{2}}^{S O}$ & $C_{L, \omega}^{S O}$ & $C_{M, \omega}^{\prime S O}$ & $C_{M, \omega^{3}}^{S O}$ \\
& 212.58 & 206.075 & 55.00 & 0.760 & -0.0018 & 26.80 & 3.864 & 0.0173 & -1193.0
\end{tabular}

TABLE 1. Critical Reynolds numbers $R e^{S S}$ and $R e^{S O}$ and values of the Archimedes number and load coefficients for $R e=R e^{S O}$ for various body shapes; $C_{M, \alpha}^{\prime S O}$ (respectively $C_{M, \omega}^{\prime} S O$ ) and $C_{x 0}^{\prime S O}$ are the derivatives of $C_{M, \alpha}$ (respectively $C_{M, \omega}$ ) and $C_{x 0}$ with respect to $R e$ evaluated at $R e=R e^{S O}$.

\& Acrivos 1993; Fabre et al. 2008; Meliga et al. 2009); the values for the other two aspect ratios agree with the results of Chrust et al. (2010).

Figure 2(a) displays the variations with the Reynolds number of coefficients $C_{L, \alpha}, C_{y, \alpha}$ and $C_{M, \alpha}$ corresponding to the loads induced by the above mode $m= \pm 1$ for a disk with an aspect ratio $\chi=10$. Not surprisingly, the loads tend to infinity as the Reynolds number approaches the critical value $R e=R e^{S S}$, and display a singularity of order one there (i.e. they diverge as $\left.\left(R e-R e^{S S}\right)^{-1}\right)$. For small enough Reynolds numbers $(R e \lesssim 105)$ the lateral projection of the force is negative $\left(C_{y, \alpha}<0\right)$ while the lift coefficient $C_{L, \alpha}$ is positive. According to figure 1, this means that the direction of the force lies in between that of the body axis and the incoming flow $(\theta>0, \gamma<0)$, as in the situation sketched in figure $1(a)$. For $105 \lesssim R e<R e^{S S} \equiv 143.9, C_{y, \alpha}$ is also positive, indicating that $\theta$ and $\gamma$ are both negative. Beyond $R e^{S S}$, both projections of the force first become negative, so that $\theta$ and $\gamma$ are both positive, as in figure $1(b)$. Then, beyond $R e \gtrsim 142, C_{L, \alpha}$ becomes positive again, leading back to the configuration encountered for $R e \lesssim 105$. The torque coefficient $C_{M, \alpha}$ is negative for $R e<R e^{S S}$. Then it becomes positive up to a critical Reynolds number, $R e^{S O}$, for which it vanishes before becoming negative again. Similar trends are observed for other aspect ratios. Table 1 provides the values of the force coefficients $C_{x 0}^{S O}$ and $C_{y, \alpha}^{S O}$ corresponding to $R e=R e^{S O}$ for various values of $\chi$. The existence of a state with non-zero incidence and zero torque for $R e=R e^{S O}$ will later prove crucial in the situation where the body is free to move, since a steady motion then implies a zero torque.

\section{4. $O\left(\alpha^{2}\right)$ and $O\left(\alpha^{3}\right)$ problems}

At order $\alpha^{2}$, the problem (2.4) and the far-field condition (2.8) take the form

$$
\mathscr{C}\left(\boldsymbol{u}_{0}, \boldsymbol{u}_{\alpha^{2}}\right)+\frac{1}{2} \mathscr{C}\left(\boldsymbol{u}_{\alpha}, \boldsymbol{u}_{\alpha}\right)+\nabla p_{\alpha^{2}}-R e^{-1} \nabla^{2} \boldsymbol{u}_{\alpha^{2}}=\mathbf{0}, \quad \nabla \cdot \boldsymbol{u}_{\alpha^{2}}=0, \quad \boldsymbol{u}_{\alpha^{2}} \rightarrow-\boldsymbol{x} / 2 .
$$

Because of the structure of the solution at order $\alpha$, the forcing term $\mathscr{C}\left(\boldsymbol{u}_{\alpha}, \boldsymbol{u}_{\alpha}\right)$ involves contributions with azimuthal wavenumbers $m=0, \pm 2$. Thus the solution at order $\alpha^{2}$ is sought in the form

$$
\boldsymbol{q}_{\alpha^{2}}=\hat{\boldsymbol{q}}_{2}^{0}+\hat{\boldsymbol{q}}_{2}^{2} \mathrm{e}^{2 \mathrm{i} \varphi}+\overline{\hat{\boldsymbol{q}}}_{2}^{2} \mathrm{e}^{-2 \mathrm{i} \varphi},
$$


where $\hat{\boldsymbol{q}}_{2}^{0}$ and $\hat{\boldsymbol{q}}_{2}^{2}$ are the respective solutions of the linear problems

$$
\begin{gathered}
\mathscr{A}_{0} \hat{\boldsymbol{q}}_{2}^{0}+\left[\mathscr{C}_{1,-1}\left(\hat{\boldsymbol{u}}_{1}^{1}, \hat{\boldsymbol{u}}_{1}^{1}\right), 0\right]^{\mathrm{T}}=0, \quad \hat{\boldsymbol{q}}_{2}^{0} \rightarrow[0,0,-1 / 2,0]^{\mathrm{T}} \quad \text { for }|\boldsymbol{R}| \rightarrow \infty, \\
\mathscr{A}_{2} \hat{\boldsymbol{q}}_{2}^{2}+\frac{1}{2}\left[\mathscr{C}_{1,1}\left(\hat{\boldsymbol{u}}_{1}^{1}, \hat{\boldsymbol{u}}_{1}^{1}\right), 0\right]^{\mathrm{T}}=0, \quad \hat{\boldsymbol{q}}_{2}^{2} \rightarrow[0,0,0,0]^{\mathrm{T}} \quad \text { for }|\boldsymbol{R}| \rightarrow \infty .
\end{gathered}
$$

Only the first of these terms contributes to the drag force and results in the drag correction $F_{x, \alpha^{2}}$ through (2.5). Note that the operators $\mathscr{A}_{0}$ and $\mathscr{A}_{2}$ are regular whatever $R e$, so that the solution at this order does not contain any new singularities, apart from those already present in the forcing terms. The solution at order $\alpha^{3}$ follows a similar route; the corresponding problem and far-field condition are

$$
\mathscr{C}\left(\boldsymbol{u}_{0}, \boldsymbol{u}_{\alpha^{3}}\right)+\mathscr{C}\left(\boldsymbol{u}_{\alpha}, \boldsymbol{u}_{\alpha}^{2}\right)+\nabla p_{\alpha^{3}}-R^{-1} \nabla^{2} \boldsymbol{u}_{\alpha^{3}}=\mathbf{0}, \quad \nabla \cdot \boldsymbol{u}_{\alpha^{3}}=0, \quad \boldsymbol{u}_{\alpha^{3}} \rightarrow \boldsymbol{x} / 6 .
$$

The solution is sought in the form

$$
\boldsymbol{q}_{\alpha^{3}}=\hat{\boldsymbol{q}}_{3}^{1} \mathrm{e}^{\mathrm{i} \varphi}+\hat{\boldsymbol{q}}_{3}^{3} \mathrm{e}^{3 \mathrm{i} \varphi}+\overline{\hat{\boldsymbol{q}}}_{3}^{1} \mathrm{e}^{-\mathrm{i} \varphi}+\overline{\hat{\boldsymbol{q}}}_{3}^{3} \mathrm{e}^{-3 \mathrm{i} \varphi},
$$

where $\hat{\boldsymbol{q}}_{3}^{1}$ is the solution of

$$
\begin{gathered}
\mathscr{A}_{1} \hat{\boldsymbol{q}}_{3}^{1}+\left[\left(\mathscr{C}_{2,-1}\left(\hat{\boldsymbol{u}}_{2}^{2}, \hat{\boldsymbol{u}}_{1}^{-1}\right)+\mathscr{C}_{0,1}\left(\hat{\boldsymbol{u}}_{2}^{0}, \hat{\boldsymbol{u}}_{1}^{1}\right)\right), 0\right]^{\mathrm{T}}=0, \\
\hat{\boldsymbol{q}}_{3}^{1} \rightarrow[1 / 12, i / 12,0,0]^{\mathrm{T}} \quad \text { for }|\boldsymbol{R}| \rightarrow \infty,
\end{gathered}
$$

which provides the lift contribution $F_{x, \alpha^{3}}$ through (2.5). The term $\hat{\boldsymbol{q}}_{3}^{3}$ does not contribute to the loads and hence does not need to be computed. All the above problems are non-singular provided $R e \neq R e^{S S}$, so that they are easily solved with a linear system solver.

The load coefficients at orders two and three are displayed in figure $2(b)$ for a disk with $\chi=10$. They are clearly highly singular for $R e \rightarrow R e^{S S}$; this is why they are plotted in logarithmic coordinates. More precisely, $C_{x, \alpha^{2}}$ exhibits a singularity of order two (since it results from the solution of a regular problem with a quadratic forcing term involving the solution at order one), while $C_{M, \alpha^{3}}$ and $C_{y, \alpha^{3}}$ exhibit a singularity of order four (since they result from the solution of a singular problem with a forcing term with a singularity of order three). Note that $C_{x, \alpha^{2}}$ and $C_{M, \alpha^{3}}$ are all positive in the range of $\operatorname{Re}$ considered, while $C_{y, \alpha^{3}}$ is negative. Similar results are obtained with other aspect ratios, except that $C_{M, \alpha^{3}}$ is found to be negative for very thin disks $(\chi \gtrsim 51)$.

The main outcomes of this section are the five coefficients $C_{x 0}, C_{y, \alpha}, C_{M, \alpha}, C_{x, \alpha^{2}}$ and $C_{M, \alpha^{3}}$ which may be used to examine the properties of non-trivial steady solutions of the full problem (2.4) close to the critical Reynolds number $R e=R e^{S O}$ at which the coefficient $C_{M, \alpha}$ provided by the solution of the problem (2.10) vanishes. The numerical values of these coefficient for $R e=R e^{S O}$, as well as other related quantities, are given in table 1 for various body geometries.

\section{Application to freely moving disks of various thicknesses}

We now turn to the situation where the body is moving freely under the effect of buoyancy. In this case, the velocity $U_{0}$ is not imposed externally and the Reynolds number is no longer a control parameter. Therefore it is convenient to introduce the socalled Archimedes number $A r=v^{-1}\left\{\left(3\left|m^{\prime}\right| g\right) /(4 \pi \rho)\right\}^{1 / 2}$, where $m^{\prime} g$ is the net gravity force (including buoyancy). Conventions for this problem are sketched in figure 1(b). Note that the angles $\gamma$ and $\theta$ defined in this figure now become the slope of the path and the body inclination with respect to the vertical, respectively. An equilibrium solution for a buoyancy-driven motion requires the torque acting on the body to be 

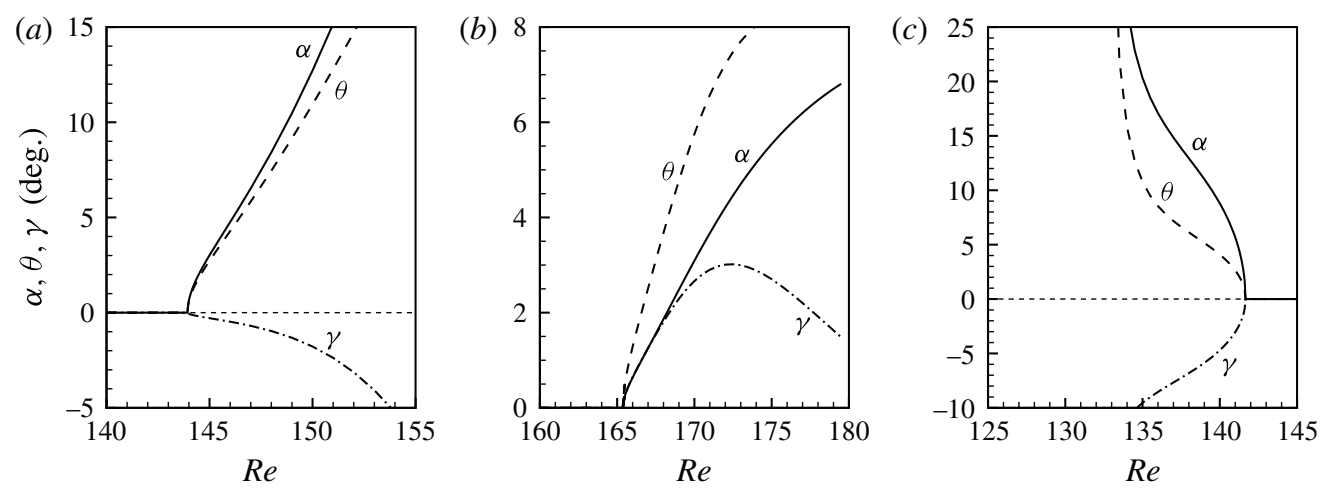

FIGURE 3. Variations with Re of the characteristic angles $\alpha, \gamma$ and $\theta$ of the weakly nonlinear solution with zero torque for: (a) $\chi=10 ;(b) \chi=3$; and (c) $\chi=\infty$.

zero and the hydrodynamic force to balance the buoyancy force. The latter condition provides a relation between $A r$ and $R e$, namely

$$
\operatorname{Ar}=(3 / 32)^{1 / 2}\left(C_{x}^{2}+C_{y}^{2}\right)^{1 / 4} \operatorname{Re} .
$$

Obviously, the axisymmetric base flow with $\alpha=\theta=\gamma=0$ is a solution of this problem. However the weakly nonlinear expansion performed in the previous section predicts a second, non-trivial type of solution. The latter, hereinafter referred to as the steady oblique (SO) solution, is obtained by requiring the torque given in (2.9) to vanish, and thus obeys the condition

$$
\alpha=\left(-M_{\alpha} / M_{\alpha^{3}}\right)^{1 / 2} .
$$

This non-trivial solution exists provided $M_{\alpha}$ and $M_{\alpha^{3}}$ have opposite signs. According to the results displayed in figure 2 and table 1, this condition is satisfied in the vicinity of $R e=R e^{S O}$. More precisely, for most aspect ratios, the SO solution exists for $R e>R e^{S O}$, indicating a supercritical bifurcation. However $M_{\alpha^{3}}$ is found to change sign for $\chi \approx 52$, so that the SO solution is found for $R e<R e^{S O}$ for very thin disks with $\chi \gtrsim 52$, indicating a subcritical bifurcation. (Note that according to figure 2, $M_{\alpha}$ and $M_{\alpha^{3}}$ also have opposite signs in the range $R e<R e^{S S}$ for $\chi=10$; however the weakly nonlinear expansion is questionable in the vicinity of $R e^{S S}$, as will be seen with the sphere, so we disregard this possibility). Imposing that the corresponding hydrodynamic force be aligned with the vertical completes the determination of the solution and provides the corresponding slope and inclination angles, namely

$$
\theta=-\tan ^{-1}\left(F_{y} / F_{x}\right) \approx \frac{\left(D_{0}-L_{\alpha}\right)}{D_{0}} \sqrt{\frac{-M_{\alpha}}{M_{\alpha^{3}}}}, \quad \gamma=-\tan ^{-1}(L / D) \approx-\frac{L_{\alpha}}{D_{0}} \sqrt{\frac{-M_{\alpha}}{M_{\alpha^{3}}}} .
$$

Figure 3 displays the three angles $\alpha, \theta$ and $\gamma$ as predicted by (3.2) and (3.3) for disks with three different aspect ratios. For $\chi=10$ (figure $3 a$ ), $R e^{S O}=143.94$ and the critical Archimedes number is found to be $A r^{S O}=46.15$ (see table 1), in very good agreement with the threshold of the oblique regime, $A r=46.5$, determined by Auguste (2010) through direct numerical simulation for a body-to-fluid density ratio of 0.99 . In this case, the slope $\gamma$ is negative, meaning that the body drifts in a direction opposite to that along which it inclines with respect to the vertical. Figure 3(b) displays the 
same result in the case of a thicker disk with $\chi=3$. In that case $\gamma$ is positive, so that the disk drifts in the direction towards which it inclines. Such opposite behaviours of thin and thick disks were observed by Fernandes et al. (2007), although at higher Archimedes numbers associated with periodic fluttering. For an infinitely thin disk $(\chi=\infty)$, figure 3(c) confirms that the bifurcation is subcritical, as could be expected from the negative sign of the coefficient $C_{M, \alpha^{3}}$ for $R e=R e^{S O}$ as indicated in table 1 . It can also be noticed that the predicted $\alpha, \gamma$, and $\theta$ blow up for $\operatorname{Re} \approx 132$, a value corresponding to a change of sign of $C_{M, \alpha^{3}}$.

To gain more insight into the nature of the bifurcation and the properties of the solution close to $R e^{S O}$, we introduce the quantity $\delta=R e-R e^{S O}$ and expand the various coefficients in series of $\delta$. For instance $C_{x 0} \approx C_{x 0}^{S O}+C_{x 0}^{\prime S O} \delta, C_{M, \alpha} \approx C_{M, \alpha}^{\prime S O} \delta$, where the prime denotes differentiation with respect to $R e$ and the superscript $S O$ indicates that the value is taken at $R e=R e^{S O}$. Note that there is no $O(1)$-term in the expansion of $C_{M, \alpha}$ owing to the definition of $R e^{S O}$. The other coefficients have similar expansions but only their leading-order value is required in what follows. The various terms involved in these expansions are obtained by linearly fitting the numerical solutions for the coefficients determined in $\S 2$ in the vicinity of $R e^{S O}$. Alternatively, they could have been derived rigorously by replacing the one-parameter expansion performed in $\S 2$ by a two-parameter expansion of the whole problem with respect to $\alpha$ and $\delta$ (we checked that this second approach, which is much more cumbersome than the one we adopted here, yields the same leading-order values for all coefficients). Introducing these expansions into (3.2) leads to

$$
\alpha \approx\left(\frac{-C_{M, \alpha}^{\prime S O}}{C_{M, \alpha^{3}}^{S O}}\right)^{1 / 2} \delta^{1 / 2}
$$

which is recognized as a standard pitchfork bifurcation equation. The coefficients entering (3.4) are given in table 1 for various values of the aspect ratio, confirming that the bifurcation is supercritical (respectively subcritical) for $\chi \lesssim 52$ (respectively $\chi \gtrsim 52$ ). Introducing the same expansions into (3.1) provides the dependence of $A r$ on $R e$ in the vicinity of the threshold as

$$
A r \approx A r^{S O}+\sqrt{\frac{3 C_{x 0}^{S O}}{32}}\left[\left(1+\operatorname{Re}^{S O} \frac{C_{x 0}^{\prime S O}}{2 C_{x 0}^{S O}}\right) \delta+\operatorname{Re} S\left(\frac{C_{x, \alpha^{2}}^{S O}}{2 C_{x 0}^{S O}}+\left\{\frac{C_{y, \alpha}^{S O}}{2 C_{x 0}^{S O}}\right\}^{2}\right) \alpha^{2}\right] .
$$

The term proportional to $\delta$ in (3.5) accounts for the dependence of the drag on the Reynolds number and is valid for both the vertical path and the SO path. The second term accounts for the additional drag resulting from the non-zero incidence along the SO path. Table 1 indicates that $C_{x, \alpha^{2}}^{S O}$ is positive, so that the whole correction to the drag due to the non-zero incidence is positive. Moreover (3.4) shows that this additional drag is actually proportional to $\delta$, leading to the conclusion that the $A r-R e$ relation displays a slope discontinuity at $R e=R e^{S O}$. Consequently, a given body (i.e. a given $A r$ ) has a lower velocity (i.e. a smaller $R e$ ) along the SO path than along a vertical path. For instance, the Reynolds number of a body with $\chi=10$ and $A r=50$ is found to be $R e \approx 150.7$ along the SO path instead of $R e \approx 159.5$ along a vertical path.

To conclude this section, it must be stressed that the properties of the SO solution, and in particular its bifurcation threshold $R e^{S O}$, are independent of the mass of the disk. Indeed, the SO regime being steady, the zero-force and zero-torque conditions are 

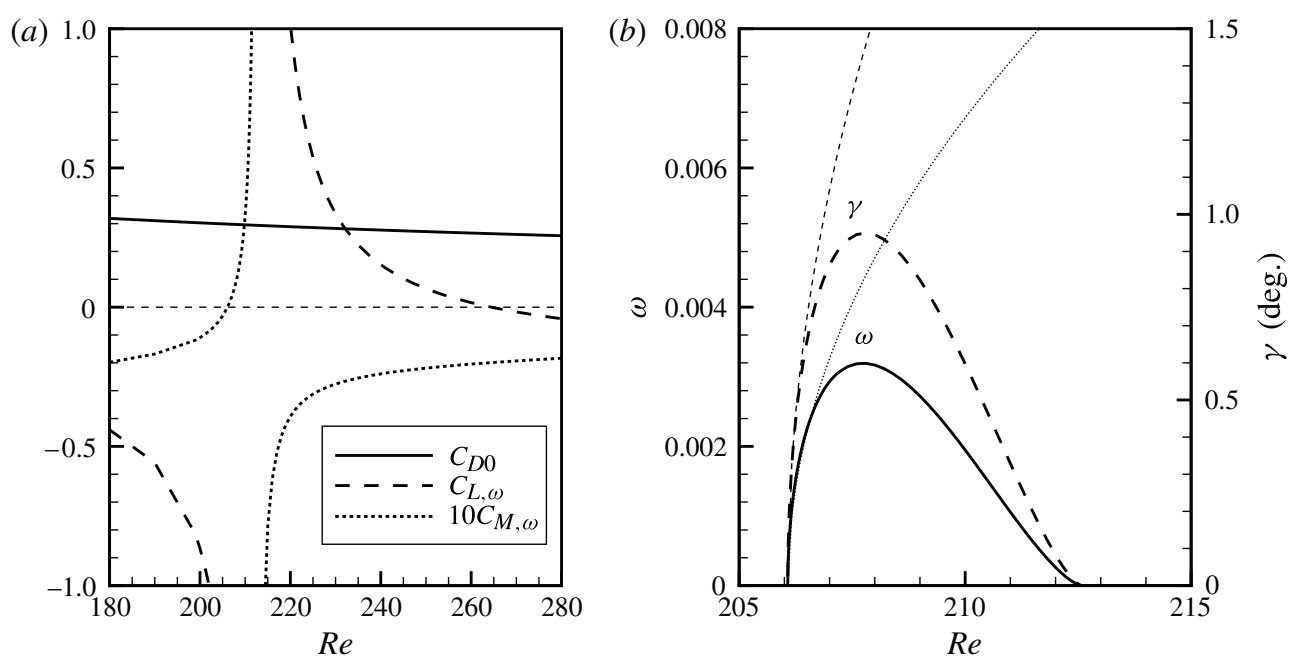

FIGURE 4. Characteristics of the SO solution for a freely moving, weakly rotating sphere. (a) Base-flow drag coefficient and order-one lift and torque coefficients; $(b)$ rotation rate $\omega$ (solid line) and slope angle $\gamma$ (dashed line); in $(b)$, the two thin lines correspond to the leading-order expansion close to $R e=R e^{S O}$.

satisfied in the absence of any acceleration of the body, be it translational or rotational. Hence, as for the axisymmetric solution corresponding to the straight vertical path, the body inertia is not involved in the SO solution, which makes its characteristics independent of the body-to-fluid density ratio.

\section{A freely moving, slowly rotating sphere}

We now turn to the case of a sphere, for which SO paths have also been reported (Jenny et al. 2004; Veldhuis \& Biesheuvel 2007; Horowitz \& Williamson 2010). In this case the rotation rate $\omega$ (made dimensionless by normalizing the actual rotation rate with $U_{0} / d$ ) takes the role of the angle of incidence. We thus seek the solution in the form

$$
\boldsymbol{q}=\boldsymbol{q}_{0}+\omega \boldsymbol{q}_{\omega}+\omega^{2} \boldsymbol{q}_{\omega^{2}}+\omega^{3} \boldsymbol{q}_{\omega^{3}}+\cdots
$$

It is now relevant to consider the problem in the aerodynamic system of axes. The corresponding boundary conditions are

$$
\boldsymbol{u}=\omega \boldsymbol{z} \times \boldsymbol{R} \text { for }|\boldsymbol{R}|=1 / 2, \quad \boldsymbol{u} \rightarrow \boldsymbol{x}_{a} \text { for }|\boldsymbol{R}| \rightarrow \infty .
$$

The successive solutions in (4.1) are computed as in $\$ 2$. The only difference lies in the boundary conditions. That is, in (4.2), the far-field condition applies to the base flow $\boldsymbol{q}_{0}$, while the no-slip condition applies to the linear correction $\omega \boldsymbol{q}_{\omega}$. For higher-order corrections, homogeneous conditions apply to $\boldsymbol{q}_{\omega^{2}}$ and $\boldsymbol{q}_{\omega^{3}}$, both on the sphere surface and in the far field. The symmetry arguments invoked in $\S 2$ still hold and so does (2.9), provided $\alpha$ is replaced by $\omega$ and $F_{x}$ and $F_{y}$ are replaced by $D$ and $L$, respectively.

Figure 4(a) displays the load coefficients predicted by the leading-order and firstorder solutions in (4.1). The torque coefficient $C_{M, \omega}$ is much smaller than the other two coefficients and is thus magnified by a factor of 10 in the figure. For Reynolds 
numbers below $R e^{S S}$, the lift coefficient is negative, i.e. the lift force points towards the direction of $\omega \boldsymbol{z} \times \boldsymbol{U}_{0}$, in accordance with the classical Kutta-Joukowski argument. However, it changes sign beyond the critical Reynolds number $R e^{S S}$ where the problem is singular and remains positive up to $R e \approx 265$ beyond which it recovers a negative sign. As for disks, the torque coefficient is found to cross zero at a single Reynolds number $R e=R e^{S O}=206.07$. However this critical Reynolds number is smaller than the fixed-body threshold $R e^{S S}=212.58$ for the sphere while the reverse was observed for disks. We shall come back to that point later. The higher-order coefficients are not displayed in the figure; as for disks they are highly singular in the vicinity of $R e^{S S}$ and $C_{M, \omega^{3}}$ is found to be negative for $R e \lesssim 245$.

As with disks, the weakly nonlinear expansion may be used to build a nontrivial steady solution of the freely moving body problem by requiring the torque in the counterpart of (2.9) to vanish. This condition may be satisfied in the range $R e^{S O}<R e<R e^{S S}$, yielding the specific value of the rotation rate $\omega=\left(-M_{\omega} / M_{\omega^{3}}\right)^{1 / 2}$. The zero-torque condition may also be satisfied for $R e>245$. However the corresponding $\omega$ is found to be of $O(1)$, so that the validity of the perturbative approach is questionable and its results are not trustworthy. The slope and inclination associated with the oblique path in the range $R e^{S O}<R e<R e^{S S}$ can be deduced from the equivalent of (3.3). The corresponding results are plotted in figure $4(b)$ which reveals that the rotation rate and the slope angle $\gamma$ are very small. The bifurcation that takes place at $R e=R e^{S O}$ is supercritical; close to the threshold one thus has at leading order $\omega \approx\left(-C_{M, \omega}^{\prime S O} / C_{M, \omega^{3}}^{S O}\right)^{1 / 2}\left(R e-R e^{S O}\right)^{1 / 2}$. The numerical values of $C_{M, \omega}^{\prime S O}$ and $C_{M, \omega^{3}}$ are given in table 1 and the corresponding leading-order predictions are plotted with thin lines in figure $4(b)$. In the vicinity of $R e=R e^{S S}$, the predicted rotation rate returns to zero, with a scaling of the form $\omega \propto\left|R e-R e^{S S}\right|^{3 / 2}$. However, as all coefficients in the expansion diverge for $R e \rightarrow R e^{S S}$, the present weakly nonlinear expansion is no longer relevant and a different approach is required to study this subregion, a point we plan to explore in the future.

The critical Archimedes number at the onset of the SO mode is given in table 1 . Instead of $A r$, Jenny et al. (2004) made use of a Galileo number defined as $2 \sqrt{2} A r$. With this definition, the critical Galileo number corresponding to the SO bifurcation is found to be 155.57, in excellent agreement with the values 155.0 and 156.1 reported by Jenny et al. for body-to-fluid density ratios of 0 and 0.5 , respectively.

It is noteworthy that $R e^{S O}<R e^{S S}$ for a sphere, whereas the reverse is observed for disks. This surprising feature may be explained with a qualitative argument based on the now well-established fact that the primary instability of axisymmetric wakes is controlled by the amount of vorticity produced at the body surface (Ern et al. 2012). In the SO configuration, a freely moving disk inclines with respect to the purely broadside configuration which, for a given incoming velocity, tends to reduce the strength of the vorticity at its surface (the more streamlined the body, the smaller the amount of vorticity produced at its surface). Therefore, one expects the wake instability in the SO state to occur at a somewhat higher Reynolds number compared to that in the SS state, which suggests $R e^{S O}>R e^{S S}$. Moreover, the larger the aspect ratio (i.e. the geometrical anisotropy of the body), the larger the influence of the disk inclination on the strength of the surface vorticity. Thus the above argument suggests that the difference $R e^{S O}-R e^{S S}$ should increase with $\chi$, and this is indeed what is observed in table 1 . In contrast, for a sphere, the slow rotation characterizing the SO state tends to increase the vorticity at the body surface for a given incoming velocity, so that one expects the instability to occur at a slightly lower Reynolds number 
compared to the strictly translational situation, which suggests $R e^{S O}<R e^{S S}$, in line with the computational results.

\section{Conclusions}

Using weakly nonlinear expansions of the Navier-Stokes equations, we predict the existence of steady oblique paths for two sorts of axisymmetric freely moving bodies, namely disks of arbitrary thickness and spheres, and describe these paths in the vicinity of the critical Reynolds number $R e^{S O}$ determined by the $C_{M, \alpha}=0$ condition. We stress that $R e^{S O}$ is the exact value of the threshold of the steady oblique path because the solution associated with that path must have zero torque, so that its branching point along the branch associated with the axisymmetric base state corresponds to a non-trivial solution of the linear problem (2.10) with a zero torque.

Since the whole derivation is carried out assuming the flow to be steady, the body inertia never enters the analysis, however large it is. As a result, the characteristics of these non-vertical paths in which the body has a constant translational and (if any) rotational velocity do not depend on the body-to-fluid density ratio. However, it is clear than when a body moves freely, the time required for its path to change from purely vertical to steady oblique certainly increases with the body inertia. Only the initial and final states of the flow are similar to those considered here in that case.

Another remarkable result of the present investigation is that the value of $R e^{S O}$ differs from that of $R e^{S S}$, the critical Reynolds number corresponding to the onset of the SS wake mode for the body held fixed. The critical Reynolds number $R e^{S O}$ has been found to be larger than $R e^{S S}$ for disks while it is smaller than the fixed-body threshold for a sphere. Although surprising at first glance, this difference may be rationalized by considering the way in which the strength of the vorticity at the body surface varies when the flow is disturbed either by a small inclination of the body (for disks) or by a slow rotation (for a sphere). It must be stressed that, while the SS wake mode and the SO path apparently have much in common, they actually correspond to two different situations: the SS mode has zero incidence and a non-zero torque (and lift), while the SO solution has zero torque and a non-zero incidence. Therefore the freely moving and fixed-body problems differ from each other and there is no reason why the solution of the former should tend toward that of the latter, even in the limit of very large body-to-fluid density ratios.

The SO solution having been obtained through an asymptotic approach, its validity when $\left|R e-R e^{S O}\right|$ increases is unknown and will have to be checked against results of full numerical simulations. An investigation based on such simulations would be of special interest in the case of the sphere, for which the weakly nonlinear method fails to predict the existence of the SO path beyond $R e^{S S} \approx 212.6$, although such paths have been reported in this range of $R e$.

Finally we must stress that the present investigation did not examine the stability of the SO solution. Recent direct numerical simulation results by Auguste (2010) show that for a specific body-to-fluid density ratio of 0.99 , the SO regime is observed (i.e. it is stable) for $\chi=10$ while it never occurs (i.e. it is unstable) for both $\chi=3$ and $\chi \rightarrow \infty$. Therefore, although the SO path is an equilibrium solution for any axisymmetric body geometry irrespective of the body-to-fluid density ratio, the stability of this solution certainly depends on this ratio. To explore this key issue, a full stability analysis of the freely moving body problem is required. We are currently investigating this problem and have already observed extra regimes with a much more 
complex dynamics than that of the steady oblique path. This will be the subject of a future article.

\section{Acknowledgements}

We thank those who conceived the FreeFem++ software for having built such a powerful tool. We also acknowledge P. Méliga, J.-M. Chomaz and D. Sipp for having designed the smart notation system used in $\S 2$, and F. Giannetti for stimulating discussions.

\section{REFERENCES}

Assemat, P., FABre, D. \& Magnaudet, J. 2012 The onset of unsteadiness of two-dimensional bodies falling or rising freely in a viscous fluid: a linear study. J. Fluid Mech. 690, 173-202.

Auguste, F. 2010 Instabilités de sillage générées derrière un corps solide cylindrique fixe ou mobile dans un fluide visqueux. PhD thesis, Université de Toulouse, France. Available online at https://www.imft.fr/Projet-ANR-OBLIC.

Chrust, M., Bouchet, G. \& Dusek, J. 2010 Parametric study of the transition scenario in the wake of oblate spheroids and flat cylinders. J. Fluid Mech. 665, 199-208.

Ern, P., Risso, F., Fabre, D. \& Magnaudet, J. 2012 Wake-induced oscillatory paths of bodies freely rising or falling in fluids. Annu. Rev. Fluid Mech. 44, 97-121.

Fabre, D., Assemat, P. \& Magnaudet, J. 2011 A quasi-static approach to the stability of the path of heavy bodies falling within a viscous fluid. J. Fluids Struct. 27, 758-767.

Fabre, D., Auguste, F. \& Magnaudet, J. 2008 Bifurcation and symmetry breaking in the wake of axisymmetric bodies. Phys. Fluids 20, 051702.

Fernandes, P. C., Risso, F., Ern, P. \& Magnaudet, J. 2007 Oscillatory motion and wake instability of freely-rising axisymmetric bodies. J. Fluid Mech. 573, 479-502.

Horowitz, M. \& Williamson, C. H. K. 2010 The effect of Reynolds number on the dynamics and wakes of freely rising and falling spheres. J. Fluid Mech. 651, 251-294.

Jenny, M., Dusek, J. \& Bouchet, G. 2004 Instabilities and transition of a sphere falling or ascending freely in a Newtonian fluid. J. Fluid Mech. 508, 201-239.

Meliga, P., Chomaz, J.-M. \& Sipp, D. 2009 Global mode interaction and pattern selection in the wake of a disk: a weakly nonlinear expansion. J. Fluid Mech. 633, 159-189.

NAtARAJAN, R. \& ACrivos, A. 1993 The instability of the steady flow past spheres and disks. J. Fluid Mech. 254, 323-344.

Veldhuis, C. H. J. \& Biesheuvel, A. 2007 An experimental study of the regimes of motion of spheres falling or ascending freely in a Newtonian fluid. Intl J. Multiphase Flow 33, 1074-1087. 